\title{
Classification of Lower Back Pain Disorder Using Multiple Machine Learning Techniques and Identifying Degree of Importance of Each Parameter
}

\author{
Abhijitsingh Putu Gaonkar, Ritwik Kulkarni, \\ Ronnie D. Caytiles* and N.Ch.S.N Iyengar** \\ School of Computer Science and Engineering, VIT University, Vellore, T.N., \\ India, \\ * Multimedia Engineering department, Hannam University, Daejeon, Korea \\ **Professor, Department of Information Technology, Sreenidhi Institute of \\ Science and Technology, Hyderabad, India \\ kchimwayi@gmail.com,noorienittoor@gmail.com,rdcaytiles@gmail.com, \\ srimannarayanach@sreenidhi.edu.in
}

\begin{abstract}
Lower back pain can occur due to various reasons involving any body part such as the interconnected network of spinal cord, nerves, bones, discs or tendons in the lumbar spine. Understanding and knowing about the origins of this disorder and getting a diagnostic treatment that determines the underlying reason, is the primary step in achieving an effective and efficient cure. Despite of heavy spending on resources such as time and money, devoted to lower back pain research methodologies, fruitful management remains a significant goal and lower back pain continues to be a cause of considerable concern on the primary care setting. One of the reason for this, could be the degrees of importance for researching into specific domain like these, are regularly developed by researchers and funding bodies, with less consideration of the needs of primary care practitioners. This study aims to determine the research priorities of primary care practitioners who manage low back pain on a day-to-day basis and to identify whether a person is abnormal or normal using collected physical spine data of 381 patients with 12 parameters. This study also identifies the degree of importance of each parameter used in the classification and ranks those parameters accordingly.
\end{abstract}

Keywords: Lower back pain, chronic disorder, Random forest classifier, SVM classifier

\section{Introduction}

Low back pain (LBP) may be a quite common disorder and affects all ranges of the population, however, its burden is commonly thought of less importance. Low back pain occurs in similar proportions in all age groups and affects with quality of life and work performance, and is the primary reason for medical consultations. Some of the cases of this disorder are due to a particular causes while most cases are non-specific. In the Global Burden of Disease Study (GBD) 2010, it was demonstrated that this disorder is one among the top ten high burden diseases and injuries, usually with higher figures in DALYs (disability-adjusted life years) higher than HIV, road injuries, tuberculosis, lung cancer, chronic obstructive pulmonary disease and preterm birth complications [1].

According to a survey conducted by 'National Center of Health Statistics', low back pain was the most common pain reported (27\%) with neck pain suffered by $15 \%$ of people, the same percentage who experience severe headaches or migraine. The report suggested that in the age groups between 45 to 64 and 65 and above, the percentage of 
this order was observed to be more than $35 \%$. The yearly occurrence of spine pain from age 5 to 65 as the lifetime occurrence can range around $84 \%$. The monthly occurrence is stated between $35 \%$ and 37\%. Many individuals have their first experiences of spine pains in late teens or early twenties and these experiences frequently reoccur throughout adult life ultimately causing severe chronic disorders. Typical reasons of low back pain include: (i) The large nerve roots in the lower back that visit the legs might be irritated, (ii) The Small nerves that supply the lower back might be irritated, (iii) The large paired spine muscles (erector spine) might be strained, (iv) The bones, ligaments or joints might be damaged.

\section{Table 1. Occurrence of Lower Back Pain in Different Age Groups}

\begin{tabular}{|c|c|c|c|}
\hline \multirow{2}{*}{$\begin{array}{c}\text { Type } \\
\text { Of } \\
\text { Disorder }\end{array}$} & \multicolumn{3}{|c|}{ Age Group (in years) } \\
\cline { 2 - 4 } & $18-44$ & $45-64$ & 65 and over \\
\hline Lower Back Pain & $23 \%$ & $35 \%$ & $37 \%$ \\
\hline Neck Pain & $12 \%$ & $20 \%$ & $15 \%$ \\
\hline
\end{tabular}

An intervertebral disc may be degenerating an irritation or trouble with some of these structures may cause spine pain and/or pain that radiates or is described as the other areas of the body. Many spine problems also cause back muscle spasms, which don't appear to be much but could cause severe pain and disability. While spine pain is very common, the observable symptoms and severity of back pain vary greatly. Following figure showcases the structure of lower spine consisting of vivid vertebral discs.

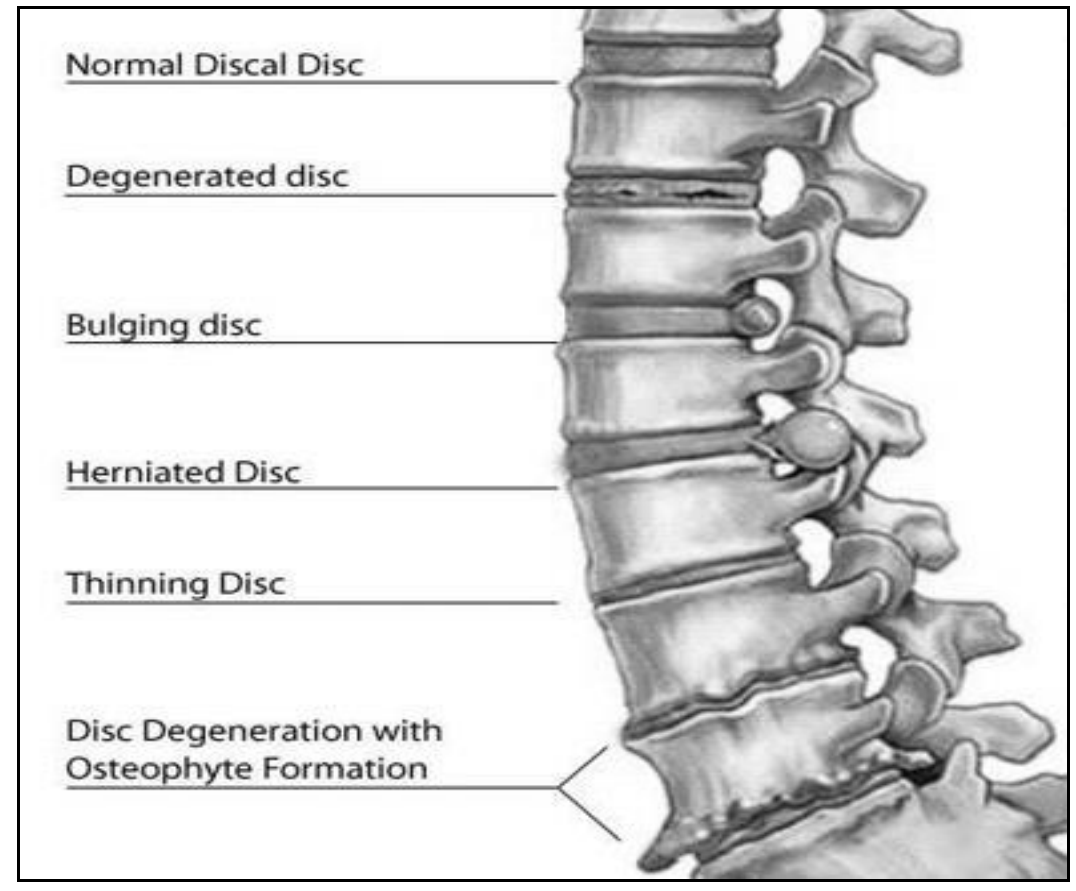

Figure 1. In-vertebral Spine Discs

Low back pain is a complex indication with many diverse causes for its presentation. There is no other part in the body that contains so many potentially pain causing structures in such a small area. This makes forming a precise diagnosis as to the tissue causing the low back pain very challenging. The formation of a medical diagnosis is imperative to enable the clinician to arrive at a suitable treatment for the pain. In $90 \%$ of 
low back pain cases, however, there is an underlying mechanical cause to the low back pain9 that will resolve itself within two to eight weeks with minimal treatment. From this it can be concluded that in a majority of cases knowledge of a definitive tissue in lesion will not dramatically effect a patient's long-term prognosis. The more important issue in the diagnosis of low back pain is differentiating these benign mechanical causes of low back pain from the more serious and pathological causes that do require immediate treatment. This particularly becomes an important consideration for manual therapists who specifically treat the mechanical causes of low back pain.

A straightforward spine muscle strain could be excruciating enough to necessitate an emergency room visit, while a degenerating disc could potentially cause only mild, intermittent discomfort. Low back pain can be due to a number of factors including: individual characteristics, working conditions such as heavy physical work, awkward static and dynamic working postures, as well as manual handling and lifting, lifestyle factors and psychological factors. A minority of cases of low back pain results from trauma to the back, osteoporosis or prolonged corticosteroid use. Relatively less common are vertebral infections, tumors and bone metastasis. The exact source of low back pain is often difficult to identify. Non-specific back pain is thus a major problem for diagnosis and treatment. Low back pain can be produced by different tissues including muscles, soft connective tissue, ligaments, joint capsules cartilage, and blood vessels. These tissues may be pulled, strained, stretched or sprained and rapidly produce an inflammation with the release of inflammatory chemicals such as cytokines and/or chemokines. These chemicals stimulate the surrounding nerve fibers resulting in the sensation of pain. Inflammatory process perpetuates the process of swelling. A reduction on blood supply to the affected area may occur so that nutrients and oxygen are not optimally delivered and removal of irritating byproducts of inflammation is impaired, creating thereby a feedback loop of inflammation and pain.

Thus it can be said that Lower Back Pain is one of the prominent factors behind health problems which requires considerable research methodologies to be formulated in order to attain a preventive approach for the health care practitioners rather than curative one. Hence, with the help of machine learning methodologies, this study aims in achieving one such preventive approach for classifying the disorder.

\section{Related Work}

In 2002, Hazel Jenkins [1], proposed an algorithm which provided a screening tool to classify lower back pain acquiescent to chiropractic treatment and pains due to pathological cause. The categorization method allow the practitioners to avoid making assumptions depending upon the heuristics and pattern recognition until it has been established that the patient is a candidate for chiropractic care. The proposed methodology considers underlying distinction of four clinical patterns which are simple mechanical low back pain, low back pain with radiculopathy, serious pathological low back pain and low back pain with psychological overlay. In the study further, an algorithmic diagnosis between these categories is considered to facilitate the evaluation of low back pain.

Later a study by Nicholas Henschke et al.[2], suggests that there exists numerous resemblances between the research questions that practitioners need answered and those promoted in clinical practice guide lines, but there are also important differences. Primary care practitioners identified a range of topics in need of further information including diagnosis, reducing the burden on primary care, and effectiveness of treatments. Their study provides important information to researchers, and highlights the importance of including primary care practitioners in the development of a research agenda.

A novel research by S. Rahimi et al. [3] is conducted using fuzzy approach for the diagnosis of Degenerative Disc Diseases. The primary focus of their study was to show the interval type-2 fuzzy hybrid rule-based system, which was the combination of forward 
and backward chaining approach in its inference engine. According to the research, combining forward and backward chaining leads to detect the exact location of degenerated disc that shows some spinal instability. The results showed that Type-2 Fuzzy Expert System could diagnose more successfully than Type-1 Fuzzy Expert System and Crisp Expert System. Using Fuzzy Expert System can decrease unnecessary cost and unnecessary people aggregation in Magnetic Resonance Imaging Centers.

In 2005, Peter O'Sullivan [4], proposed a research study which used maladaptive movement and motor control impairments as underlying mechanism for the diagnosis and classification of chronic low back pain (CLBP) disorders. The study proposed existence of three groups of CLBP disorders, among which first group of disorders presents underlying pathological processes driving the pain, and the patient's motor responses in the disorder are adaptive. A second group of disorders present where psychological and/or social factors represent the primary mechanism underlying the disorder that centrally drives pain, and where the patient's coping and motor control strategies are maladaptive in nature. Finally it is proposed that there is a large group of CLBP disorders where patients present with either movement impairments (characterized by pain avoidance behavior) or control impairments (characterized by pain provocation behavior). In the study further, it is also proposed that there is a large sub-group of CLBP disorders where mal-adaptive movement and control impairments dominate the disorder, resulting in either excessive or impaired dynamic spinal stability and loading. This in turn becomes a mechanism for ongoing pain. Physiotherapy interventions that are classification based and specifically directed to the underlying driving mechanism, have the potential to alter these disorders and impact on both the primary physical and secondary cognitive drivers of pain. This approach is not limited only to the lumbo-pelvic region but can be applied to all regions of the musculoskeletal system.

\section{Proposed Work}

The study addresses the classification of lower back pain data consisting of 12 parameters related to the vertebral lower spine using machine learning techniques and compares the obtained results. The parameters used for classification are pelvic incidence, pelvic tilt, lumbar lordosis angle, sacral slope, pelvic radius, degree of spondylolisthesis, pelvic slope, direct tilt, thoracic slope, cervical tilt, sacrum angle and scoliosis slope. The work is carried out in a step by step manner forming a basis for concrete algorithm. Since algorithms provide an excellent screening tool for general categorization, their use has been criticized as too limited in a definitive diagnosis of low back pain. They can be used effectively to screen a patient for their general category of low back pain presentation. Furthermore, algorithms provide an easily reproducible and time effective method of ensuring that no potentially serious signs and symptoms are missed. This approach provides reliable method of ensuring that their patients receive the most appropriate care. The algorithm used is depicted in the following figure which consists of the stages of the work. 


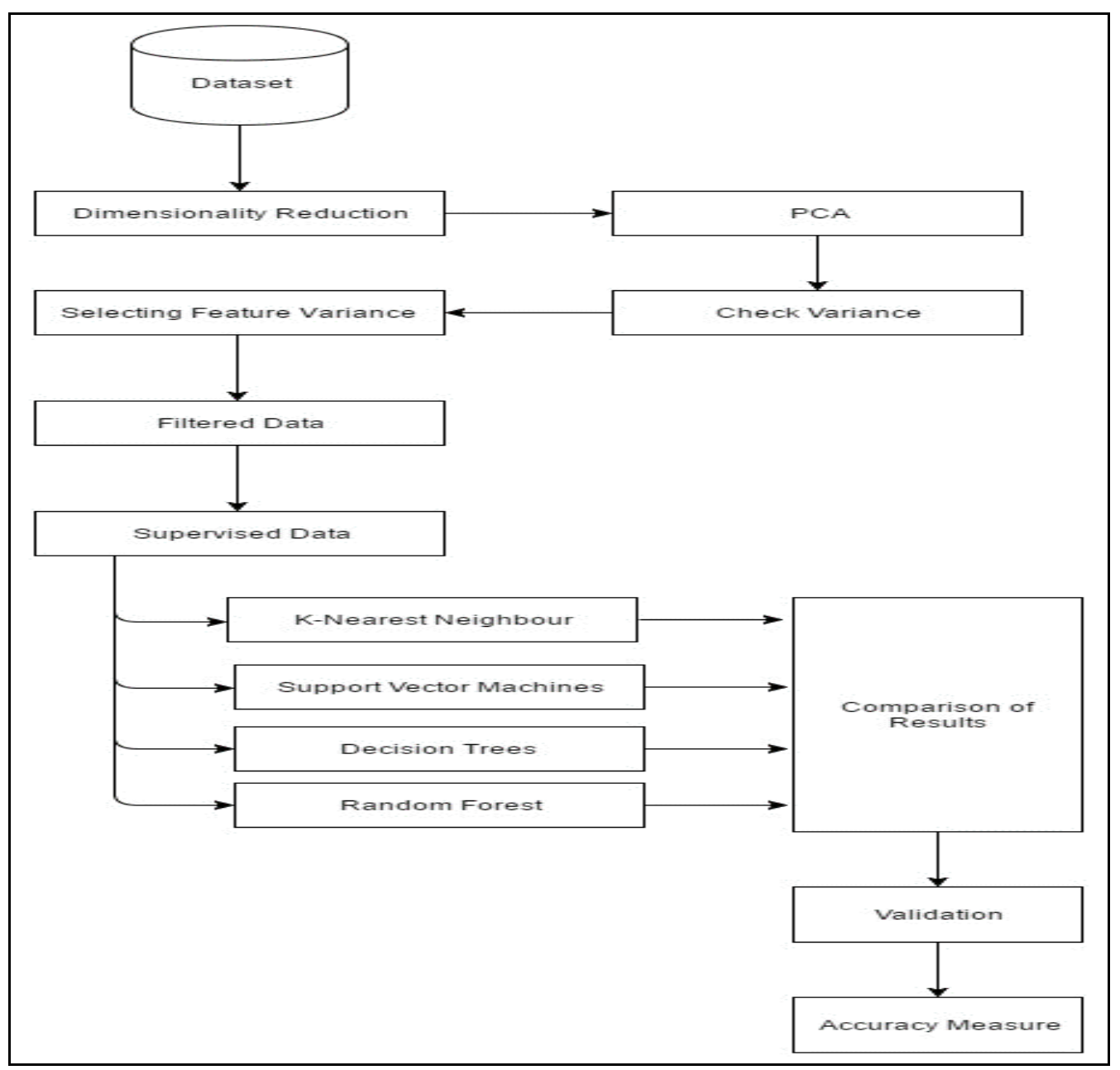

Figure 2. Algorithmic Flowchart

In the initial stage, Principal Component Analysis (PCA) is applied on dataset for feature selection. But in specific terms, it is specifically used to find possible outliers. PCA is a statistical procedure that uses an orthogonal transformation to convert a set of observations of possibly correlated variables into a set of values of linearly uncorrelated variables called principal components (or sometimes, principal modes of variation). The number of principal components is less than or equal to the smaller of the number of original variables or the number of observations. This transformation is defined in such a way that the first principal component has the largest possible variance (that is, accounts for as much of the variability in the data as possible), and each succeeding component in turn has the highest variance possible under the constraint that it is orthogonal to the preceding components. The resulting vectors are an uncorrelated orthogonal basis set. PCA is sensitive to the relative scaling of the original variables.

Mathematically, the transformation is defined by a set of p-dimensional vectors of weights or loadings,

$$
w_{(k)}=\left(w_{1}, \ldots, w_{p}\right)_{k}
$$

that map each row vector $\mathrm{x}_{\mathrm{i}}$ of $\mathrm{X}$ to a new vector of principal component

$$
t_{(i)}=\left(t_{1}, \ldots, t_{m}\right)_{i}
$$

, given by 


$$
t_{k(i)}=x_{i}, w_{k} \quad \text { for } \quad i=1, \ldots, n \quad k=1, \ldots, m
$$

In such a way that the individual variables of $t$ considered over the data set successively inherit the maximum possible variance from $\mathrm{x}$, with each loading vector $\mathrm{w}$ constrained to be a unit vector. In this case the p-dimensional vectors is the values of all 12 attributes of dataset converged into individual vectors to be treated as weights. Loading the first vector $\mathrm{w}_{1}$ has to satisfy

$$
w_{(1)}=\arg \max _{\|w\|} \mid=1\left\{\sum_{i=1}\left(t_{1}\right)_{(i)}^{2}\right\}=\arg \max _{\|w\|=1} \sum_{i=1}\left(x_{i}, w\right)^{2}
$$

Since $\mathrm{w}_{1}$ has been defined to be a unit vector, it equivalently also satisfies,

$$
w_{(1)}=\arg \max \left\{\frac{w^{T} X^{T} X_{W}}{w^{T} w}\right\}
$$

Finally, the dimensionality reduction is obtained by mapping the transformation $\mathrm{T}=\mathrm{XW}$ from original space of $\mathrm{p}$ variables to a new space of $\mathrm{p}$ variables which are uncorrelated over the dataset. Keeping only the first L principal components, produced by using only the first L loading vectors, gives the truncated transformation

$$
T_{L}=X W_{L}
$$

After applying PCA, the detected outliers are eliminated and the filtered data is considered for further process of analysis.

Once the filtered dataset is obtained with minimal covariances among the vectors, supervised learning techniques are applied on it. Supervised learning is the machine learning task of inferring a function from labeled training data. The training data consist of a set of training examples. In supervised learning, each example is a pair consisting of an input object (typically a vector) and a desired output value (also called the supervisory signal). A supervised learning algorithm analyzes the training data and produces an inferred function, which can be used for mapping new examples. An optimal scenario will allow for the algorithm to correctly determine the class labels for unseen instances. This requires the learning algorithm to generalize from the training data to unseen situations in a reasonable way.

In pattern recognition, the k-nearest neighbor algorithm $(\mathrm{k}-\mathrm{NN})$ is a non-parametric method used for classification and regression. In both cases, the input consists of the $\mathrm{k}$ closest training examples in the feature space. The output depends on whether k-NN is used for classification or regression. In k-NN classification, the output is a class membership. An object is classified by a majority vote of its neighbors, with the object being assigned to the class most common among its $\mathrm{k}$ nearest neighbors ( $\mathrm{k}$ is a positive integer, typically small). If $\mathrm{k}=1$, then the object is simply assigned to the class of that single nearest neighbor. In k-NN regression, the output is the property value for the object. This value is the average of the values of its $\mathrm{k}$ nearest neighbors. $\mathrm{k}-\mathrm{NN}$ is a type of instance-based learning, or lazy learning, where the function is only approximated locally and all computation is deferred until classification. The k-NN algorithm is among the simplest of all machine learning algorithms used in this study.

The k-nearest neighbor classifier can be viewed as assigning the $\mathrm{k}$ nearest neighbors a weight $1 / \mathrm{k}$ and all others 0 weight. This can be generalized to weighted nearest neighbor classifiers. That is, where the $\mathrm{i}^{\text {th }}$ nearest neighbor is assigned a weight $\mathrm{w}_{\mathrm{ni}}$ with

$$
\sum_{\mathrm{Z} n=1}^{i=1} w_{n i}=1
$$


An analogous result on the strong consistency of weighted nearest neighbor classifiers also holds.

Further in this study, Support Vector Machine (SVM) and Random Forest techniques are used for classification and to identify the degree of importance of each parameter and also to analyze low back pain (LBP) data. In machine learning, support vector machines are supervised learning models with associated learning algorithms that analyze data used for classification and regression analysis. This model is based on statistical learning theory (Vapnik, 1995) and attempts to search an optimal hyper-plane as a decision function in high dimensional space. The SVMs use structural risk minimization, over empirical risk minimization. Empirical risk reduces the misclassification error on the training set, whereas structural risk reduces the probability of misclassifying a previously unseen data point drawn randomly from a fixed but unknown probability distribution (Lin, 2005).

Assume, the training data with $\mathrm{k}$ number of samples be interpreted by $\left\{\mathrm{x}_{\mathrm{i}}, \mathrm{y}_{\mathrm{i}}\right\}, \mathrm{i}=1 \ldots \mathrm{k}$, where $x \in R^{N}$, which is a $N$ - dimensional space and $y \in\{-1,+1\}$ is the class label. These training patterns are told to be linearly separable if there exists a vector $\mathrm{w}$ (determining the orientation of a discriminating plane) and a scalar b (determine the offset of the discriminating plane from the origin) such that

$$
y_{i}\left(w \cdot x_{i}+b\right)-1 \geq 0
$$

The hypothesis space can be defined by the set of functions given by

$$
f_{w, b}=\operatorname{sign}(w \cdot x+b)
$$

A number of kernel functions can be used with SVMs. Vapnik (1995) discussed kernels and the parameters of the kernel function in detail. SVMs were initially designed for binary (two-class) problems. When dealing with multiple classes, a "one against one" approach (Knerr et al, 1990) is used in this study.

Random forests or random decision forests are an ensemble learning method for classification, regression and other tasks, that operate by constructing a multitude of decision trees at training time and outputting the class that is the mode of the classes (classification) or mean prediction (regression) of the individual trees. Random decision forests correct for decision trees' habit of over fitting to their training set.

These are the algorithms to be followed for random forest:

- Decision tree learning

- Tree bagging

- From bagging to random forests

- Extra Trees

Decision trees are one of the most famously used methods for various machine learning problems. In detail, the trees that are grown very deep are to learn highly irregular patterns. They have the low bias, but very high variance for their training set. This boosts the performance of the model.

The tree bagging algorithm for the random forests applies for bootstrap aggregating or bagging to tree learners. Given a training set $\mathrm{X}$, where,

$$
X=x_{1}, \ldots, x_{n}
$$

With corresponding to set $\mathrm{Y}$, where,

$$
Y=y_{1}, \ldots \ldots, y_{n}
$$

Which when bagging is chooses continuously a random sample with replacement of the training set and fits to the samples. 
In from bagging to random forests, the given procedure describes the original bagging algorithm for trees. In random forests, they use a modified tree learning algorithm that chooses when each candidate split in the learning process, a random subset of the features. Typically, for a classification problem with $p$ features, $\sqrt{ } \mathrm{p}$ (rounded down) features are used in each split.

Extra trees is one further step of randomization which gets extremely randomized trees or Extra trees which are trained using bagging and the random subspace method which more like random forests. Here, a random value is chosen and is split and the value is selected from features empirical range

\section{Results and Findings}

As stated earlier, the initial stage involved of performing PCA on the dataset, results achieved can be seen in the following figure,

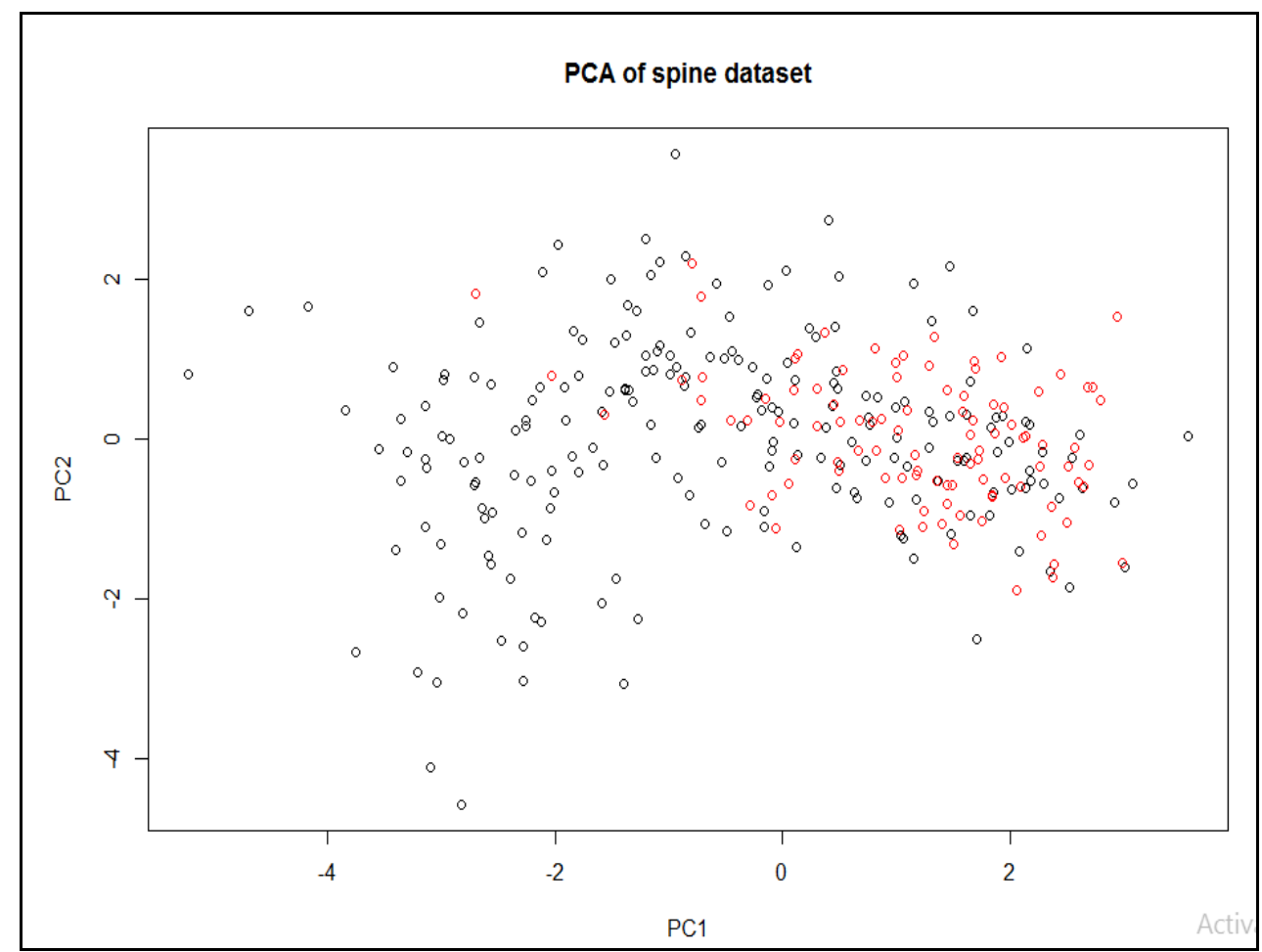

Figure 3. Result of PCA Applied on LBP Dataset

Here, the dataset is plotted in a p-dimensional space consisting of 2 weighted classes (normal, black colored dots and abnormal, red colored dots). Applying PCA on the dataset gave one outlier consisting of values which contradicted the values present in the rough sets. The final dataset consisted of 311 records, each record representing data for individual patient consisting of selective parameters. The results of classification were obtained by applying 4 machine learning techniques and the class value attribute for each record holds either of the value, 'normal' or 'abnormal' depending upon the rough sets generated. The 'abnormal' class value indicates that the degree of spondylolisthesis parameter is beyond the defined thresholds. This indirectly suggests that patient's condition is 'severe' and requires a prior spinal inspection.

Interestingly by applying classification methodologies such as Random Forest, decision trees and k-nearest neighbor, the most significant parameter always resulted to be is the degree of spondylolisthesis based on its p-value eval(1.303923e-35). Spondylolisthesis is the forward or anterior displacement of one vertebra over another, and commonly 
involving the fifth lumbar vertebra. Backward displacement is referred to as retrolisthesis. When occurring in conjunction with scoliosis, the shortened term "olisthesis," may sometimes be used instead. This implies that while classifying the lower back pain disorder, the degree of spondylolisthesis is most required parameter followed by pelvic incidence and lumbar lordosis angle.

By applying $t$ test of hypothesis on the selective parameters, the $p$-values of individual parameters is as follows,

Table 2. Degrees of Importance of Data Parameters

\begin{tabular}{|c|c|c|c|}
\hline Parameter name & p-value & Parameter name & p-value \\
\hline degree_spondylolisthesis & $1.303923 \mathrm{e}-35$ & cervical_tilt & $4.911099 \mathrm{e}-01$ \\
\hline pelvic_incidence & $1.892358 \mathrm{e}-11$ & pelvic_slope & $1.000000 \mathrm{e}+00$ \\
\hline pelvic_tilt & $1.258929 \mathrm{e}-10$ & Direct_tilt & $1.000000 \mathrm{e}+00$ \\
\hline lumbar_lordosis_angle & $7.502388 \mathrm{e}-10$ & thoracic_slope & $1.000000 \mathrm{e}+00$ \\
\hline pelvic_radius & $1.570032 \mathrm{e}-09$ & sacrum_angle & $1.000000 \mathrm{e}+00$ \\
\hline sacral_slope & $2.329476 \mathrm{e}-04$ & scoliosis_slope & $1.000000 \mathrm{e}+00$ \\
\hline
\end{tabular}

The inference corresponds to a forward displacement of a vertebral bone in relation to the natural curve of the spine. The other significant parameters $(\mathrm{p}$-value $<0.05)$ are the pelvic incidence ( $\mathrm{p}$-value $=1.892358 \mathrm{e}-11)$, pelvic tilt $(\mathrm{p}$-value $=1.258929 \mathrm{e}-10)$, lumbar lordosis angle ( $\mathrm{p}$-value $=7.502388 \mathrm{e}-10)$, pelvic radius ( $\mathrm{p}$-value $=1.570032 \mathrm{e}-09)$, and sacral slope ( $\mathrm{p}$-value $=2.329476 \mathrm{e}-04)$. The significant variables were used as predictors to classify normal and abnormal spines. A simple random forest classifier gave reasonable sensitivity (85-90\%), but poor specificity (65-70\%). Such performance could be improved with additional, more balanced data and with more sophisticated classification algorithms. Altogether, these results are initial steps towards an automatic discrimination of normal and abnormal spines, which may assist practitioners in the clinical treatment of lower back pain.

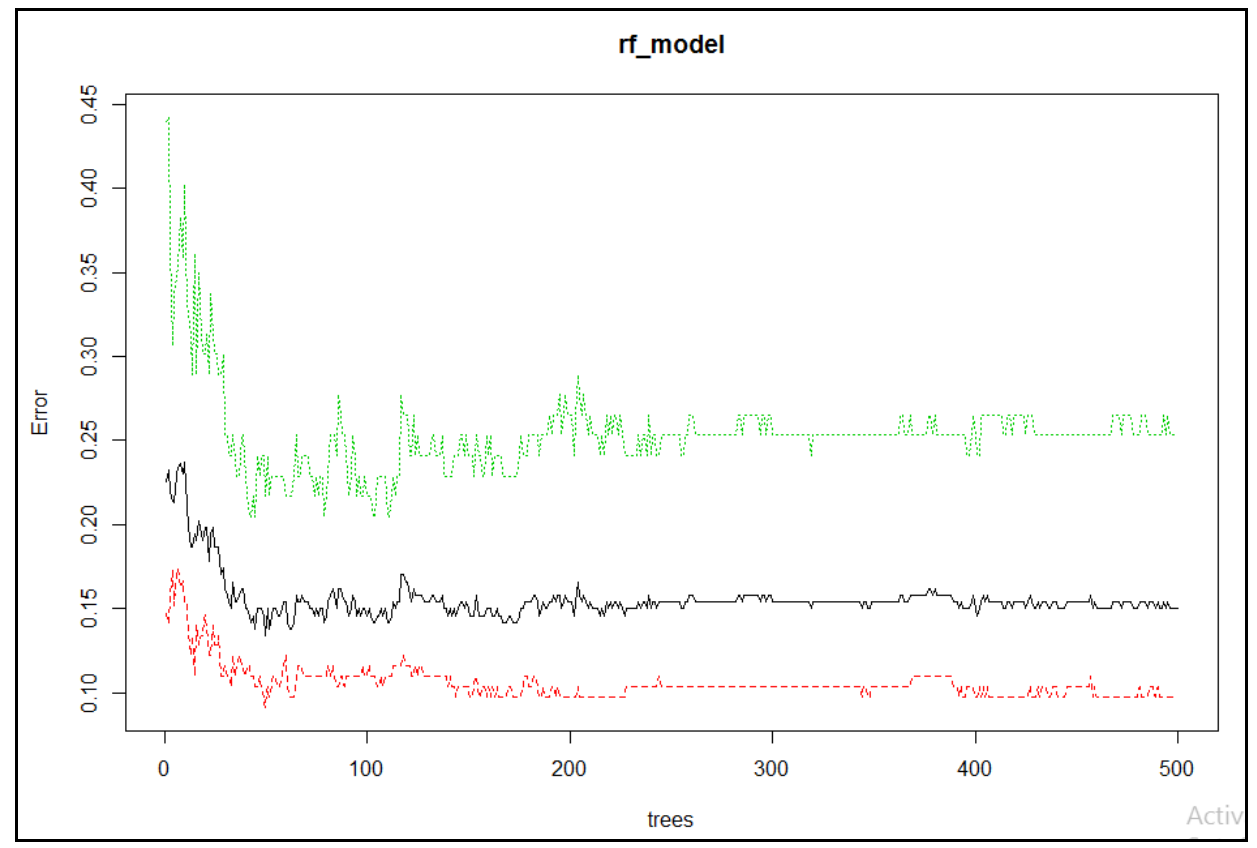

Figure 4. Random Forest Error Vs Trees 
Out-of-bag (OOB) error, also called out-of-bag estimate, is a method of measuring the prediction error of random forests, boosted decision trees, and other machine learning models utilizing bootstrap aggregating to sub-sample data samples used for training. OOB is the mean prediction error on each training sample $\mathrm{x}_{\mathrm{i}}$, using only the trees that did not have $x_{i}$ in their bootstrap sample. Subsampling allows one to define an out-of-bag estimate of the prediction performance improvement by evaluating predictions on those observations which were not used in the building of the next base learner. Out-of-bag estimates help avoid the need for an independent validation dataset, but often underestimate actual performance improvement and the optimal number of iterations.

Furthermore, following figure showcases the results obtained by decision tree wherein all the parameters associated with $\mathrm{p}$-value are represented as tree.

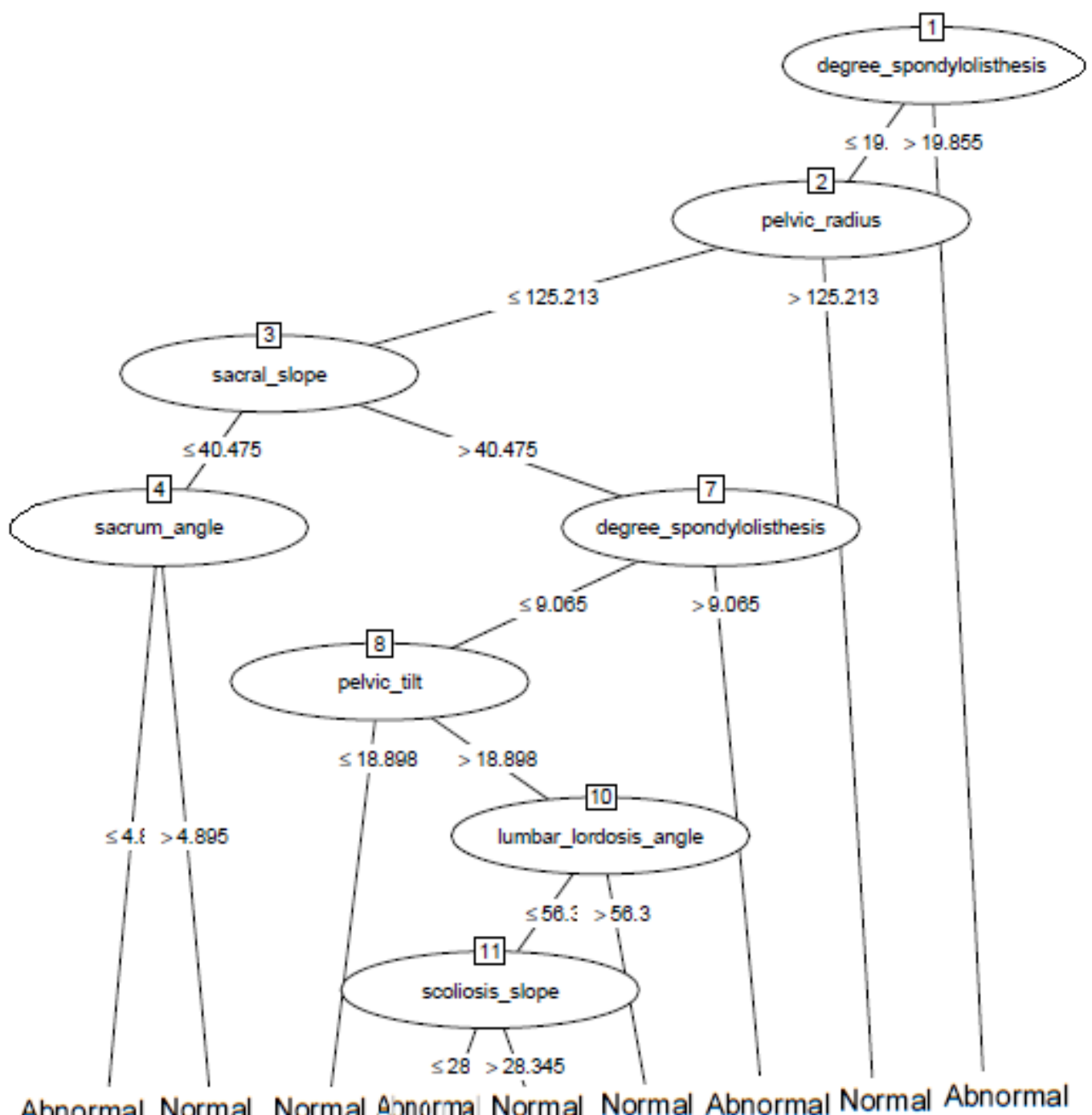

Figure 5. Results of Decision Tree

Accuracy ratios of 4 machine learning methodologies were collected and it was observed that $\mathrm{J} 48$ decision tree algorithm yields a highest accuracy ratio (90.30\%) followed by Random Forest (87.09\%). The following table shows the accuracy ratios of each method in percentages. 
Table 3. Accuracy Comparison of Machine Learning Techniques

\begin{tabular}{|c|c|}
\hline Classifier & Accuracy Rate \\
\hline k-nearest neighbor & 85.48387 \\
\hline RandomForest & 87.09677 \\
\hline J48 Decision Tree & 90.29126 \\
\hline Support Vector Machine & 85.48387 \\
\hline
\end{tabular}

After applying classification methods, the results of degrees of importance of each parameter can be seen in the following figure.

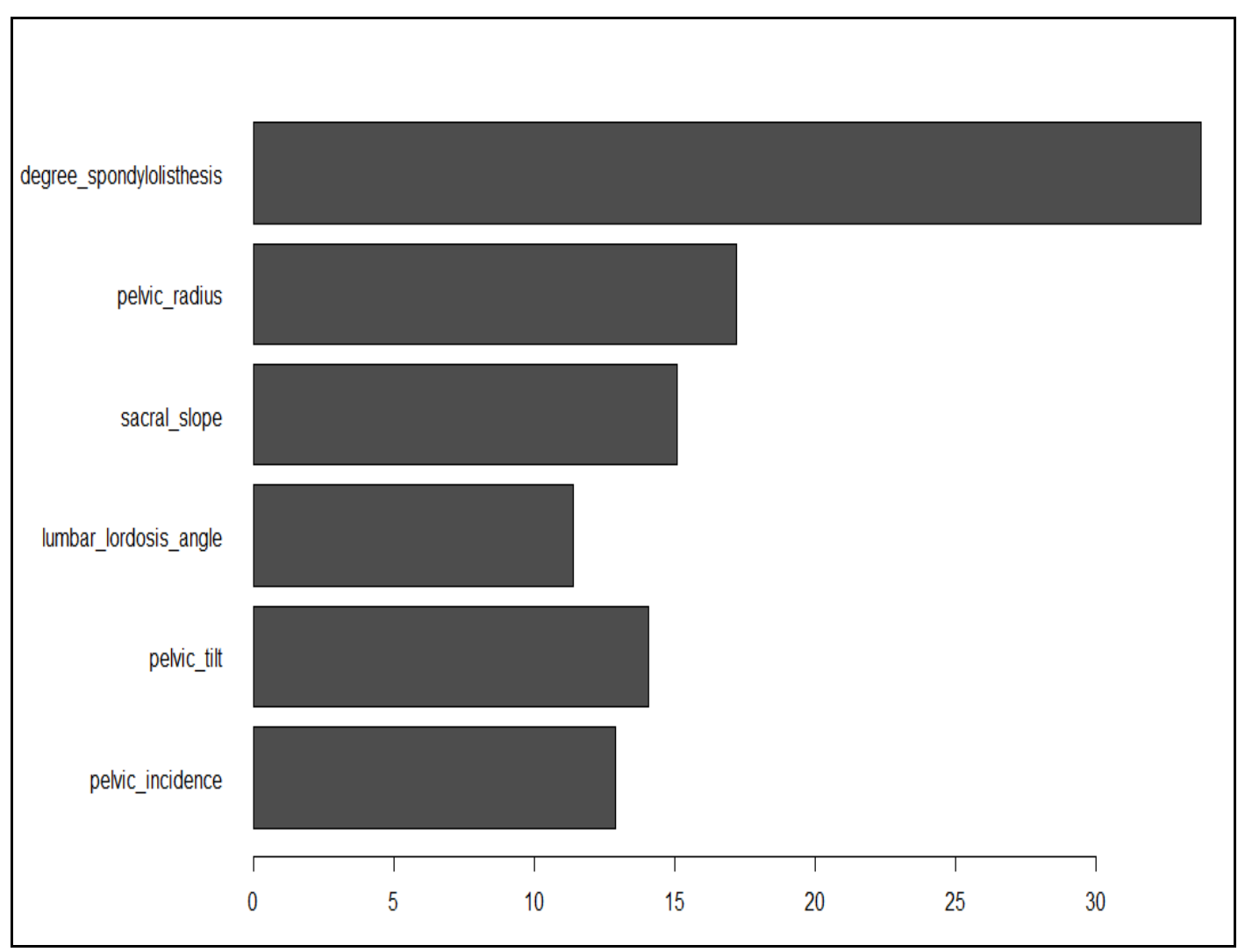

Figure 6. Levels of Importance for LBP Parameters

\section{Conclusion}

Back pain is not a disease but a constellation of symptoms which origins remain in most cases unknown even though risks factors have been identified. Low back pain is disabling and causes enormous socioeconomic impacts on societies. Treatments for now are focused on reducing the pain. Back pain is both a major cause of temporary disability and a challenge to medical and surgical treatment decisions. It imposes high socio-economic burden in modern western countries, since it not only affects the elderly population but also the working population from the age group 25-60 years. In this study, we have demonstrated classification of lower back pain in to two classes based on selective parameters. Furthermore, degrees of importance of each parameter is discussed. 


\section{Acknowledgements}

The author Dr. N.Ch.S.N.Iyengar is thankful to Dr. P.Narasimha Reddy, Executive Director, SNIST and Dr. G. Viswanathan, Chancellor, VITU for their support and encouragement.

\section{References}

[1] B. Duthey, "Background Paper 6.24 Low back pain", (2013).

[2] H. Jenkins, "Classification of low back pain", Australasian Chiropractic \& Osteopathy, vol. 10, no. 2 (2002), p. 91.

[3] N. Henschke, R.W.J.G. Ostelo, M. W. van Tulder, J.W.S. Vlaeyen, S. Morley, W.J.J. Assendelft, and C. J. Main. "Behavioural treatment for chronic low-back pain", The Cochrane Library, (2010).

[4] S. Rahimi Damirchi-Darasi, M.H. Fazel Zarandi and M. Izadi, "Type-2 Fuzzy Hybrid Expert System For Diagnosis Of Degenerative Disc Disease", Amirkabir University of Technology (Tehran Polytechnic), vol. 45, no. 2, (2013), pp. 53-62.

[5] P. O. Sullivan, "Diagnosis and classification of chronic low backpain disorders: Maladaptive movement and motor control impairments as underlying mechanism", Body-logic Physiotherapy, 146 Salvado Rd, Wembley, WA 6014, Australia School of Physiotherapy, Curtin University of Technology, Perth, Western Australia, (2005).

[6] M. Sari, E. Gulbandilar, and A. Cimbiz, "Prediction of low back pain with two expert systems," J Med Syst, vol. 36, (2012), pp. 1523-7.

[6] J. Koh, V. Chaudhary, E. K. Jeon, and G. Dhillon, "Automatic spinal canal detection in lumbar MR images in the sagittal view using dynamic programming", Computerized Medical Imaging and Graphics, vol. 38, (2014), pp. 569-579.

[7] R. Alomari, J. Corso, V. Chaudhary, and G. Dhillon, "Lumbar Spine Disc Herniation Diagnosis with a Joint Shape Model", Computational Methods and Clinical Applications for Spine Imaging. vol. 17, J. Yao, T. Klinder, and S. Li, Eds., ed: Springer International Publishing, (2014), pp. 87-98.

[8] J. Koh, V. Chaudhary, and G. Dhillon, "Disc herniation diagnosis in MRI using a CAD framework and a two-level classifier," Int Journal of Comput Assist Radiol Surg, vol. 7, (2012), pp. 861-9.

[9] S. Ghosh, R. S. Alomari, V. Chaudhary, and G. Dhillon, "Composite features for automatic diagnosis of intervertebral disc herniation from lumbar MRI", Conf Proc IEEE Eng Med Biol Soc, (2011), pp. 506871.

[10] S. Ghosh, R. S. Alomari, V. Chaudhary, and G. Dhillon, "Computer-aided diagnosis for lumbar mri using heterogeneous classifiers," Biomedical Imaging: From Nano to Macro, 2011 IEEE International Symposium, (2011), pp. 1179-1182.

[11] R. S. Alomari, J. J. Corso, V. Chaudhary, and G. Dhillon, "Computer-aided diagnosis of lumbar disc pathology from clinical lower spine MRI," Int J Comput Assist Radiol Surg, vol. 5, (2010), pp. 287-93.

\section{Authors}

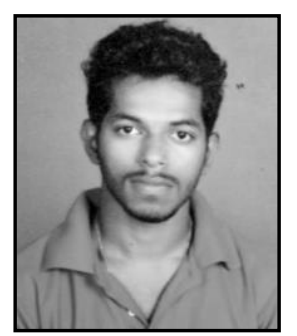

Abhijitsingh Putu Gaonkar, currently pursuing M.tech in Computer Science and Engineering with specialization in Big Data Analytics from University, Vellore Institute of Technology(V.I.T) batch of 2016-18. I have completed my B.E in Computer Engineering in 2015 from PCCE, Goa. His research interests include Machine Learning, Deep Learning and Spatial Data Mining in Healthcare.

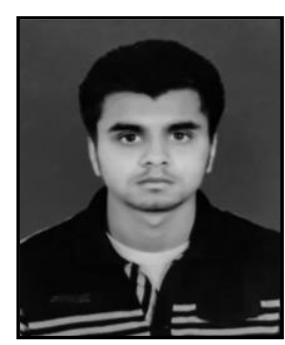

Ritwik Kulkarni, Currently pursuing M.tech in Computer Science and Engineering specialization in Big Data Analytics from University, Vellore Institute of Technology(V.I.T) batch of 2018. He completed B.E in 2016 from P.V.P.I.T, Pune. His research interests include advanced analytics using big data technologies. 


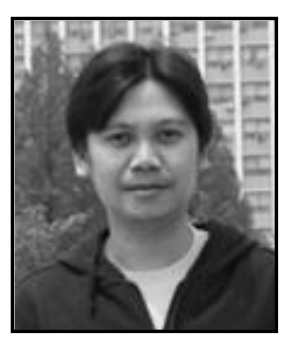

Ronnie D. Caytiles, he had his Bachelor of Science in Computer Engineering- Western Institute of Technology, Iloilo City, Philippines, and Master of Science in Computer Science- Central Philippine University, Iloilo City, Philippines. He finished his Ph.D. in Multimedia Engineering, Hannam University, Daejeon, Korea. Currently, he serves as an Assistant Professor at Multimedia Engineering department, Hannam University, Daejeon, Korea. His research interests include Mobile Computing, Multimedia Communication, Information Technology Security, Ubiquitous Computing, Control and Automation

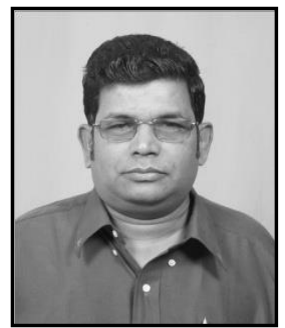

N. Ch. S. N. Iyengar (b 1961), currently Professor, Information Technology, Sreenidhi Institute of Science (SNIST) and Technology, Yamnapet, Ghatkesr, Hyderabad-501301, Telengana, India $\mathrm{He}$ is a doctorate in both Applied Mathematics and Computer Science and Engineering. His research interests include Agent-Based Distributed Computing, Intelligent Computing, Network Security, Cloud Computing, Big Data Analytics and Fluid Mechanics. He had 32+ years of experience in teaching to B.Tech. and M.Tech students. He guided 12 Ph.Ds ,5 M.Phils and $75+$ M.Tech Projects apart from authoring several textbooks. He had 200+ research publications in reputed peer reviewed international journals along with students. He organized many conferences/workshops and continuing education programmes. He served as Keynote speaker/ / Invited speaker //PCM/reviewer for many International conferences. He serves as a Editor in chief/Guest editor /Editorial board member for many international journals. He is the professional member of many bodies. 
International Journal of Advanced Science and Technology Vol.105 (2017) 